\title{
ESTIMATIVA DE RADIAÇÃO SOLAR VIA MODELAGEM ATMOSFÉRICA DE MESOESCALA APLICADA À REGIÃO NORDESTE DO BRASIL
}

\author{
OTACILIO LEANDRO DE MENEZES NETO ${ }^{1}$, ALEXANDRE ARAÚJO COSTA ${ }^{2}$ E FERNANDO PINTO \\ RAMALHO ${ }^{2}$
}

\author{
${ }^{1}$ Fundação Cearense de Meteorologia e Recursos Hídricos (FUNCEME) Fortaleza - CE \\ ${ }^{2}$ Universidade Estadual do Ceará, Fortaleza - CE
}

otacilio@funceme.br, alexandrearaujoc@gmail.com, fpramalho@gmail.com

Recebido Março 2008 - Aceito Janeiro 2009

\section{RESUMO}

A utilização de fontes alternativas de energias, como a solar, a eólica e a biomassa, vem crescendo significativamente nos últimos anos, sendo a energia solar, em particular, uma fonte abundante na região Nordeste do Brasil. O conhecimento preciso da radiação solar incidente é, assim, de grande importância para o planejamento energético brasileiro, servindo de base para o desenvolvimento de futuros projetos de plantas fotovoltaicas e de aproveitamento da energia solar. Este trabalho apresenta uma metodologia para o mapeamento da energia solar incidente ao nível do solo para a região Nordeste do Brasil, utilizando um modelo atmosférico de mesoescala (Regional Atmospheric Modeling System - RAMS), validado e ajustado por meio dos dados medidos pela rede de plataformas de coleta de dados (PCDs) da Fundação Cearense de Meteorologia e Recursos Hídricos (FUNCEME). Os resultados mostraram que o modelo apresenta erros sistemáticos, sobreestimando a radiação na superfície, porém após as devidas correções estatísticas, utilizando-se uma relação entre a fração de cobertura de nuvens prevista pelo modelo e a radiação observada na superfície e estimada no topo da atmosfera, encontram-se correlações de 0,92 com intervalos de confiança de $13,5 \mathrm{~W} / \mathrm{m}^{2}$ para dados com base mensal. Usando essa metodologia, a estimativa do valor médio anual (após ajustes) da radiação solar incidente no estado do Ceará é de $215 \mathrm{~W} / \mathrm{m}^{2}$ (máximo em outubro: $260 \mathrm{~W} / \mathrm{m}^{2}$ ).

Palavras-chave: Energia Solar, Transferência Radiativa, Modelagem Numérica.

\begin{abstract}
ESTIMATION OF SOLAR RADIATION BY MESOSCALE ATMOSPHERIC MODELING APPLIED TO THE NORTHEAST BRAZIL REGION.

The use of renewable energy sources, like solar, wind and biomass is rapidly increasing in recent years, with solar radiation being a particularly abundant energy source over Northeast Brazil. Thus, the proper quantitative knowledge of the incoming solar radiation is of great importance for energy generation planning in Brazil, serving as basis for developing future projects of photovoltaic power plants and solar energy exploration. This work presents a methodology for mapping the incoming solar radiation at ground level on Northeast Brazil, using a mesoscale atmospheric model (Regional Atmospheric Modeling System - RAMS), calibrated and validated using the automatic surface stations network data from the Ceará State Foundation for Meteorology and Water Resources (Fundação Cearense de Meteorologia e Recursos Hídricos- FUNCEME). The results showed that the model presents systematic errors, overestimating surface radiation, but, after a proper statistical corrections, using a relationship between the model-predicted cloud fraction and the observed radiation at ground level and the estimated at the top of the atmosphere, a 0.92 correlation value, with a confidence interval of $13,5 \mathrm{~W} / \mathrm{m}^{2}$, is obtained for monthly data. Using this methodology, an estimated $215 \mathrm{~W} / \mathrm{m}^{2}$ annual average incoming solar radiation over Ceará is found (maximum in October: $260 \mathrm{~W} / \mathrm{m}^{2}$ ).
\end{abstract}

Keywords: Solar Energy, Radiative Transfer, Numerical Modeling. 


\section{INTRODUÇÃO}

A energia que recebemos do Sol é indispensável para a vida na Terra, pois ela é responsável por diversos processos que ocorrem em nosso planeta, desde a fotossíntese realizada pelas plantas até os fenômenos atmosféricos. Esta energia pode também ser aproveitada para gerar energia elétrica, através de painéis fotovoltaicos, ou para aquecimento.

Na região Nordeste do Brasil é abundante a quantidade de energia solar e sua conversão em energia elétrica deve ser estimulada, o que certamente contribuirá para poupar a água armazenada nos reservatórios das usinas hidroelétricas, permitindo o seu uso para fins mais nobres, já que a região citada sofre bastante com problemas de escassez de água.

Por estes motivos, o mapeamento da radiação solar incidente no estado do Ceará e na sua extensão para a região Nordeste como um todo, é de extrema importância para o planejamento energético brasileiro. Desse modo, isto permite que projetos de instalação de plantas fotovoltaicas e de aproveitamento de energia solar pelas mais diversas tecnologias, possam ser efetuados em uma base sólida, que reduza os riscos econômicos e estratégicos para os investidores ou o próprio governo.

Tal mapeamento já vem sendo feito pelo Centro de Previsão de Tempo e Estudos Climáticos / Instituto Nacional de Pesquisas Espaciais (CPTEC/INPE) (www.cptec.inpe.br) através do modelo GL 1.2, que utiliza imagens do satélite GOES para estimar a radiação solar incidente para toda a América do Sul. Entretanto, esta metodologia não permite uma previsão das quantidades da radiação, apenas a "observação" via satélite. Acredita-se, que com o constante desenvolvimento dos modelos regionais, tal previsão seja possível.

Neste sentido, uma metodologia alternativa é aqui proposta utilizando-se o modelo de mesoescala RAMS 6.0 na sua última versão 6.0 (Pielke et al., 1992, Cotton et al., 2003).

Com a utilização dos dados coletados nos últimos anos pelas mais de 70 estações meteorológicas (Plataformas de Coleta de Dados - PCDs) operadas pela Fundação Cearense de Meteorologia e Recursos Hídricos (FUNCEME), usados na fase de validação do modelo, pôde-se estabelecer com boa precisão o mapeamento da radiação solar incidente sobre a região Nordeste do Brasil, através de ajustes nos resultados do modelo RAMS baseados nas medidas reais obtidas das PCDs.

\section{DADOS E METODOLOGIA}

Foram realizados testes de sensibilidade no modelo RAMS, a fim de escolher a melhor configuração para os cálculos da radiação solar incidente. Durante as simulações foi mantida a mesma configuração do modelo, exceto pela mudança da parametrização de radiação.

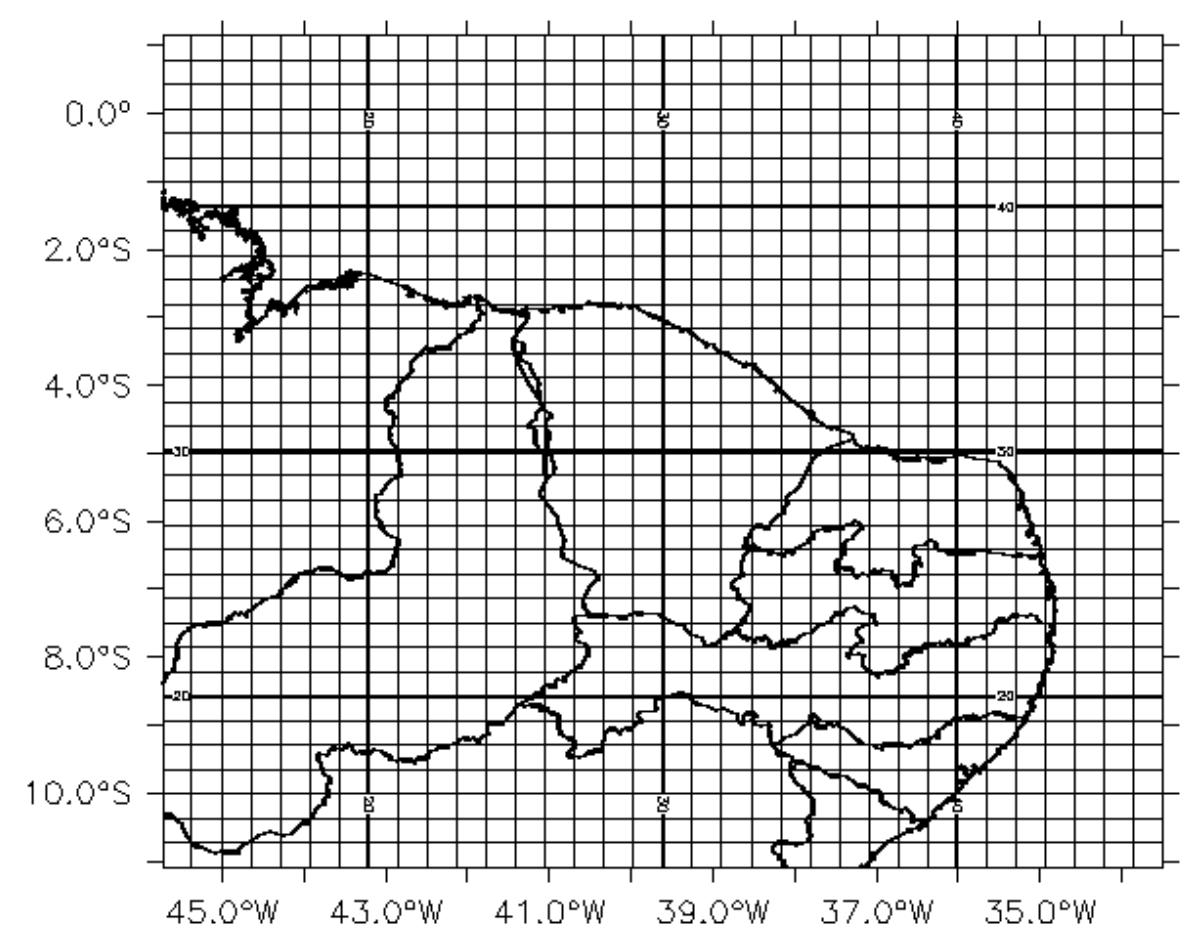

Figura 1 - Grade horizontal usada nas simulações do presente trabalho. 
O ano de 2005 (janeiro a dezembro) foi escolhido devido à disponibilidade dos dados observados pelas PCDs. As condições iniciais e forçantes de grande escala foram fornecidas pelos dados de reanálise do NCEP/NCAR, com assimilação utilizando Relaxamento Newtoniano de 1200s nas laterais, 43200s no topo e 86400 s no centro da grade. A grade horizontal continha 60 pontos em cada direção, com $40 \mathrm{~km}$ de espaçamento, cobrindo quase todo o Nordeste do Brasil (Figura 1). Utilizou-se este espaçamento de grade horizontal de $40 \mathrm{~km}$ devido ao alto custo computacional, caso fossem escolhidas

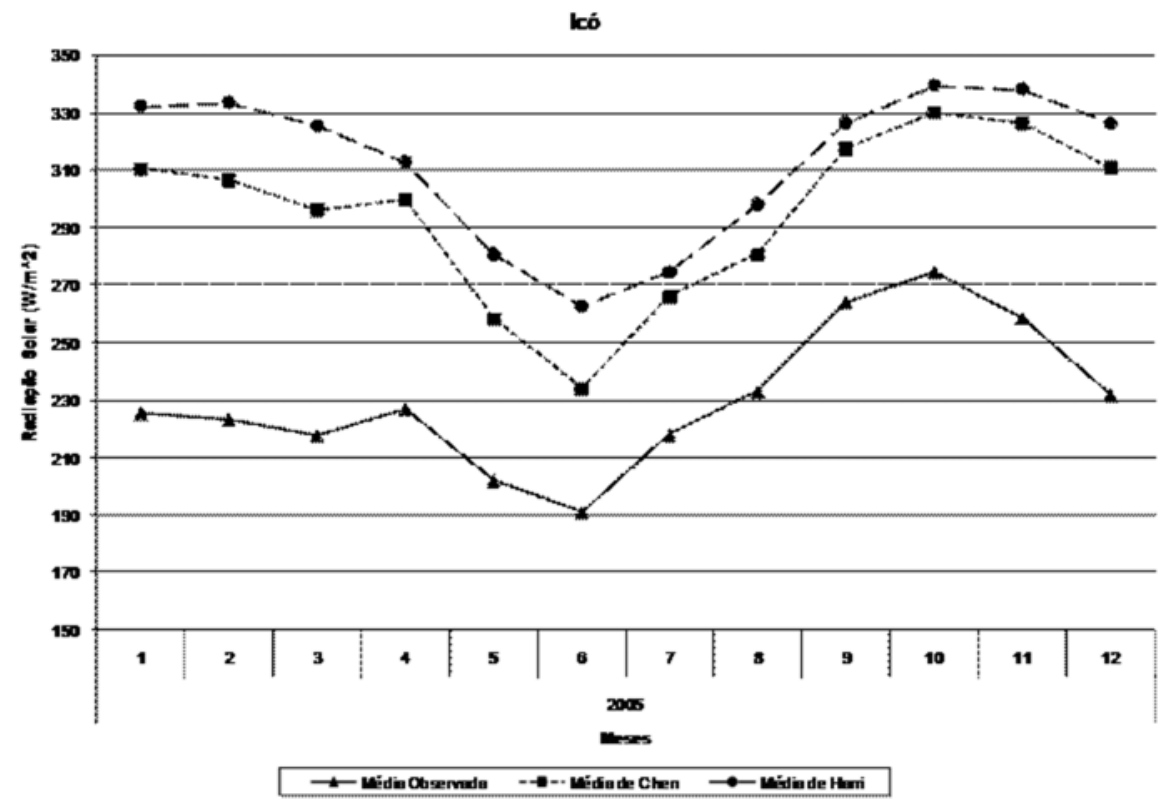

a)

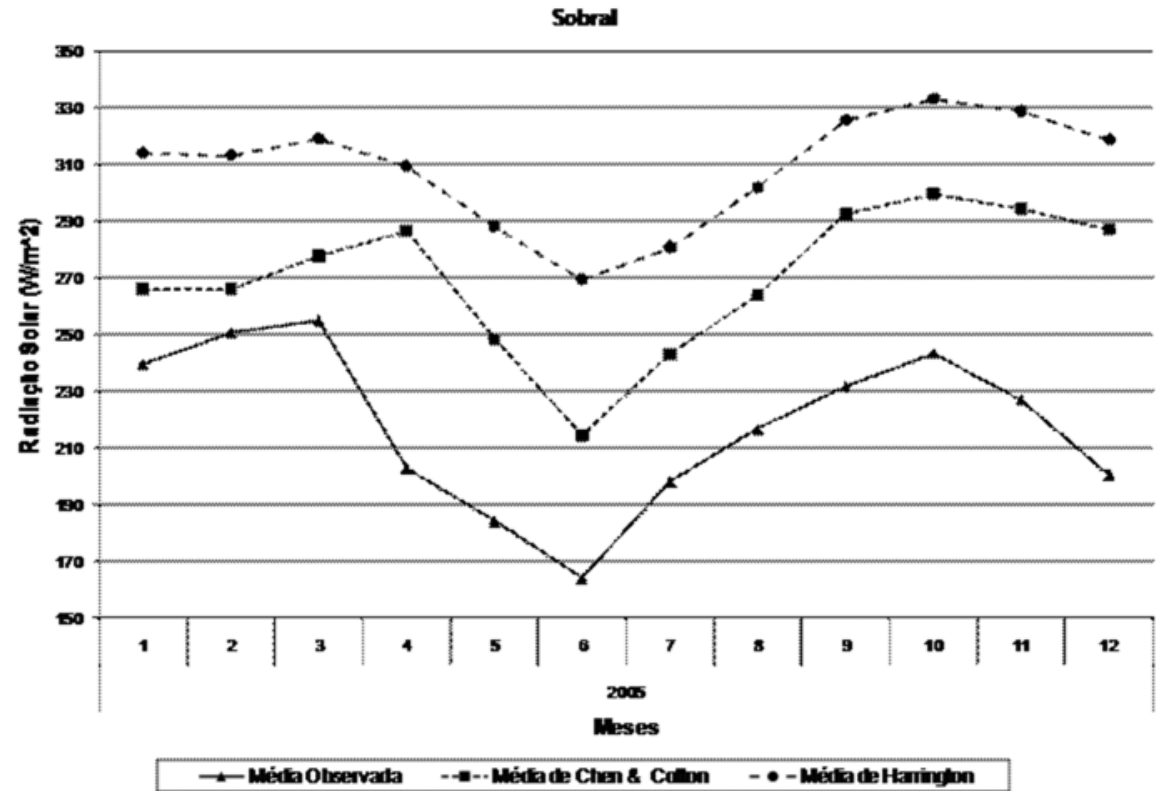

b)

Figura 2 - Comparação entre as simulações e as PCDs para as localidades de (a) Icó e (b) Sobral. 
grades mais finas. A grade vertical continha 39 níveis, com espaçamento de grade de $80 \mathrm{~m}$ próximo à superfície, razão de expansão de 1,10 , espaçamento máximo de $1000 \mathrm{~m}$ e topo a aproximadamente $24 \mathrm{~km}$. As principais parametrizações utilizadas foram as de convecção (Kuo), turbulência (MellorYamada), microfísica (Walko et al., 1995) e radiação (variável testada: Harrington, 1997 versus Chen-Cotton, 1983). A radiação foi calculada pelo modelo a intervalos de 10 minutos (por serem computacionalmente muito dispendiosos) e os resultados foram armazenados a cada hora de simulação.

\section{RESULTADOS}

Para a configuração utilizada no modelo, descrita no item 2, a parametrização de Chen-Cotton mostrou resultados mais consistentes.

Uma especulação para que a parametrização de Harrington não tenha tido sucesso nesta simulação, deve-se ao fato da resolução horizontal e vertical utilizada não ser muito alta, não sendo assim possível para o modelo resolver com precisão a convecção e, conseqüentemente, a interação da microfísica com a radiação, necessária para este esquema. Possivelmente, em simulações com grades mais finas haja uma melhoria significativa dos resultados para o esquema de radiação de Harrington, hipótese esta que ainda precisa ser testada.

Uma comparação entre os dois tipos de parametrização utilizados e os dados observados é mostrada na Figura 2, para duas localidades no interior do estado do Ceará. Devido á falta de destreza (skill) do modelo para resolver a radiação solar em uma base diária, uma alternativa foi utilizar a média mensal, calculada utilizando às 24 horas do dia ao longo dos 30 dias do mês.
Nos dois casos (e nos demais não apresentados aqui), a parametrização de Chen-Cotton apresentou resultados mais próximos às medidas observadas pelas $\mathrm{PCDs}$, embora com erros ainda consideráveis. Por este motivo esta será a parametrização utilizada, porém alguns ajustes se fazem necessários, a fim de minimizar os erros sistemáticos do modelo.

Como foi mostrado na Figura 2, o resultado do modelo possui um erro sistemático, sempre superestimando os valores da radiação solar incidente. Dois fatores mais prováveis que levam o modelo a apresentar estes erros sistemáticos são: a resolução espacial ser grossa a ponto que o modelo não consiga estimar corretamente a convecção responsável pela formação de nuvens e a maneira como o modelo "interpreta" as nuvens (através de uma parametrização utilizando a umidade relativa, temperatura potencial e pressão).

Para ajustar os resultados do modelo às observações, foram feitas algumas regressões simples para tentar encontrar o melhor ajuste que se aproximasse dos resultados observados. A regressão mais simples primeiramente testada foi uma regressão linear cuja formula é:

$$
\operatorname{Rad}=(\text { rshort } 129,33) / 0,7132
$$

em que Rad é a radiação solar corrigida e rshort é o nome da variável para a radiação solar de onda curta no modelo RAMS, ou seja, o resultado bruto do modelo. Após o uso dessa correção, a correlação entre estimativas do modelo e observações, numa base mensal, é de 0,7614 , com erro quadrático médio de $21,33 \mathrm{~W} / \mathrm{m}^{2}$, que são valores razoáveis. O motivo pelo qual essa correção não ter sido utilizada na estimativa final da radiação é que ela subestima os valores

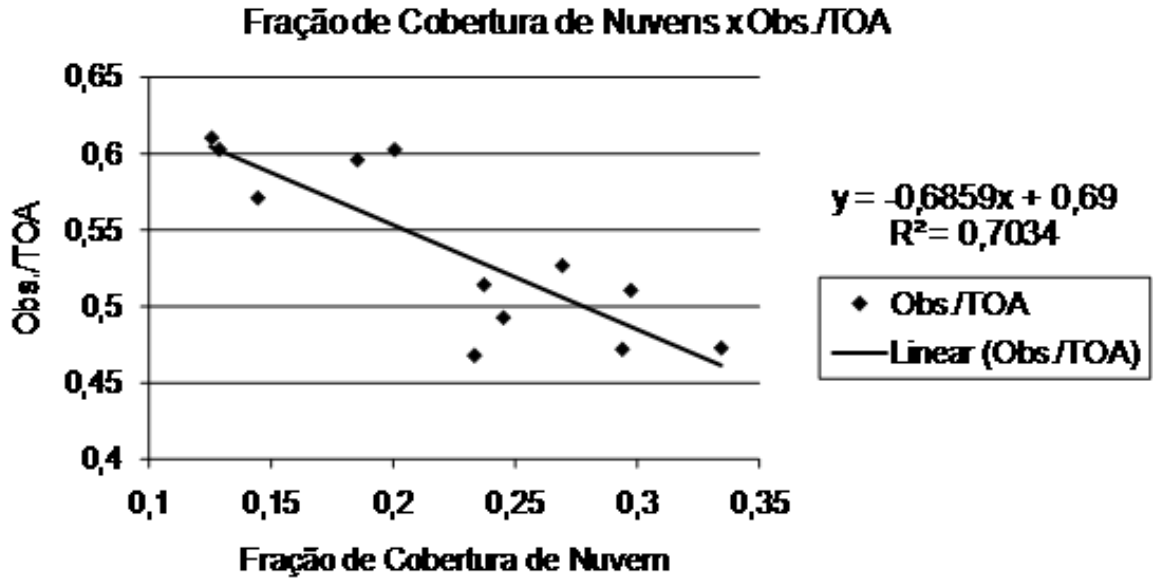

Figura 3 - Regressão linear entre a fração de cobertura de nuvem e a razão entre a radiação observada / radiação no topo da atmosfera. 
mínimos, devido ao termo independente $(-129,33)$ na equação (1). Por exemplo, a estimativa mensal para o mês de Junho de 2005 usando a equação (1) foi de somente $80 \mathrm{~W} / \mathrm{m}^{2}$, muito abaixo da média observada.

Outra relação simples testada foi uma lei de potência, dada por:

$$
\text { Rad }=0,7109 \text { rshort }{ }^{1,0141}
$$

Esta relação de potência, pelo fato de não possuir um termo independente como na relação linear, se mostra mais adequada, mas não se percebeu melhoria na correlação $(0,7615)$ apesar da redução no erro quadrático médio para 16,39 W/m2. Várias outras relações foram testadas, como por exemplo, relações logarítmicas e polinomiais de várias ordens, mas sem apresentar melhorias significativas nos resultados.

Verificou-se então, que existe uma forte relação entre a fração de cobertura de nuvens calculada pelo modelo e a razão entre a radiação observada na superfície e no topo da atmosfera
(TOA, sigla em inglês) (Guarnieri et al., 2007), como mostrado na Figura 3. O modelo RAMS calcula a fração de cobertura de nuvem como sendo a porcentagem da área de cada caixa de grade usada na simulação que está coberta por nuvens. A radiação no topo da atmosfera (TOA) foi calculada através da fórmula:

$$
T O A=S \cdot\left(\frac{A U}{r}\right)^{2} \cdot \cos z
$$

em que $\mathrm{S}$ é a constante solar, AU é a unidade astronômica, r é a distância "instantânea" Terra-Sol e cos z é o co-seno do ângulo zenital do Sol.

A função empírica que relaciona estas variáveis é:

$$
\text { Rad }=(-0,6859 \text { Nuvem }+0,69) \text { TOA }
$$

Esta relação (que foi apelidada de "cloud") apresenta melhorias significativas na correlação $(0,9219)$ e também no

Tabela 1 - Comparação estatística entre correções ao modelo.

\begin{tabular}{|l|cc|c|}
\hline \multicolumn{1}{|c|}{ Ajuste } & $\mathrm{R}$ & $\mathrm{R}^{2}$ & Erro Quadrático Médio (W/m²) \\
Observado x Rshort (sem ajuste) & 0,7614 & 0,5797 & 67,7 \\
\hline Observado x Correlação Linear & 0,7614 & 0,5797 & 21,3 \\
\hline Observado x Correção por Lei de Potência & 0,7615 & 0,5896 & 16,4 \\
\hline Observado x "Cloud" & 0,9219 & 0,7034 & 12,0 \\
\hline
\end{tabular}

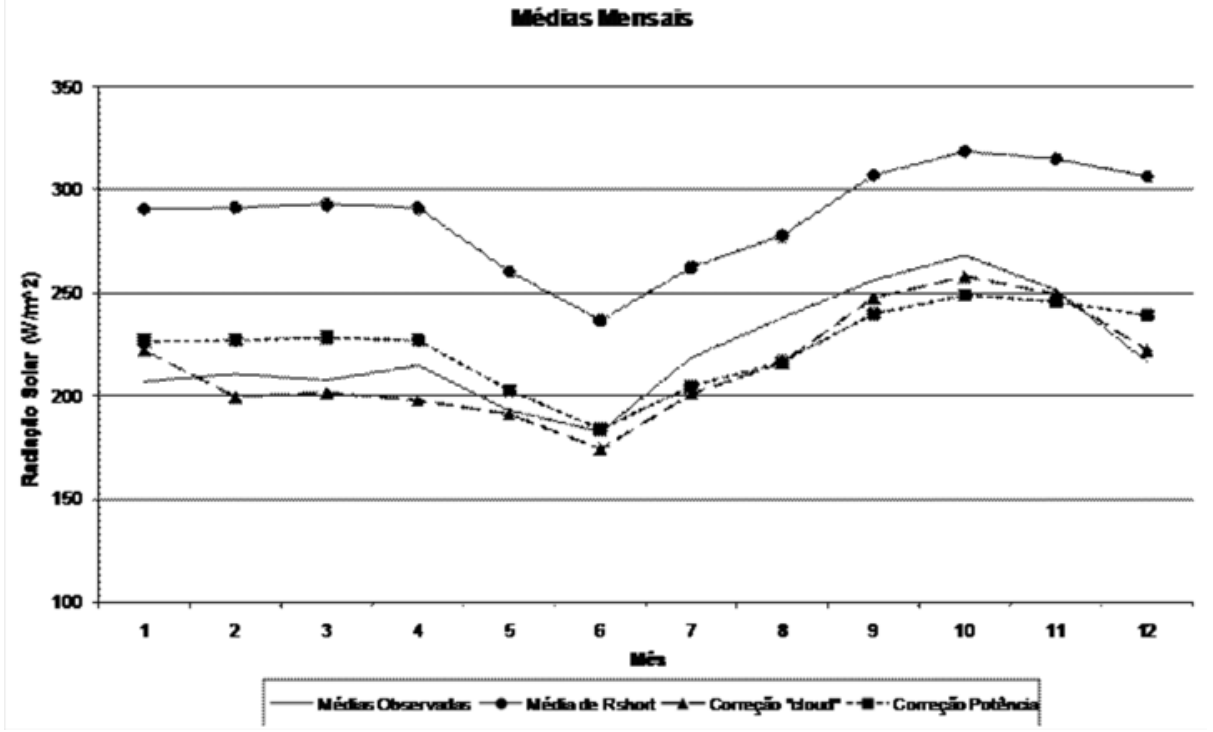

Figura 4 - Comparação entre as radiações observadas, modeladas sem ajustes e ajustadas. 
erro quadrático médio $\left(12,0 \mathrm{~W} / \mathrm{m}^{2}\right)$ sendo assim escolhida como o melhor ajuste para a radiação solar calculada pelo modelo. Um breve resumo das correções estatísticas testadas é mostrado na Tabela 1.

Na Figura 4 tem-se uma comparação entre os ajustes propostos e os dados observados, considerando-se a média sobre uma área contendo 14 PCDs com dados contínuos para o período simulado, calculada usando o método de Thiessen. Note que os dois ajustes, tanto o de potência quanto o ajuste "cloud", aproximam-se bastante da curva observada. O intervalo de confiança para o ajuste "cloud" é de $\pm 13,5 \mathrm{~W} / \mathrm{m}^{2}$.

Para verificar se $\mathrm{o}$ ajuste aqui proposto tem confiabilidade, apresenta-se na Figura 5 a distribuição de freqüência dos erros absolutos entre os dados observados e o ajuste "cloud".

Sendo o ajuste utilizando a fração de cobertura de nuvem o que apresentou melhores correlações e menor erro quadrático médio, a Figura 6 apresenta a média anual para o ano de 2005 sobre o território de parte do Nordeste do Brasil. Os valores de

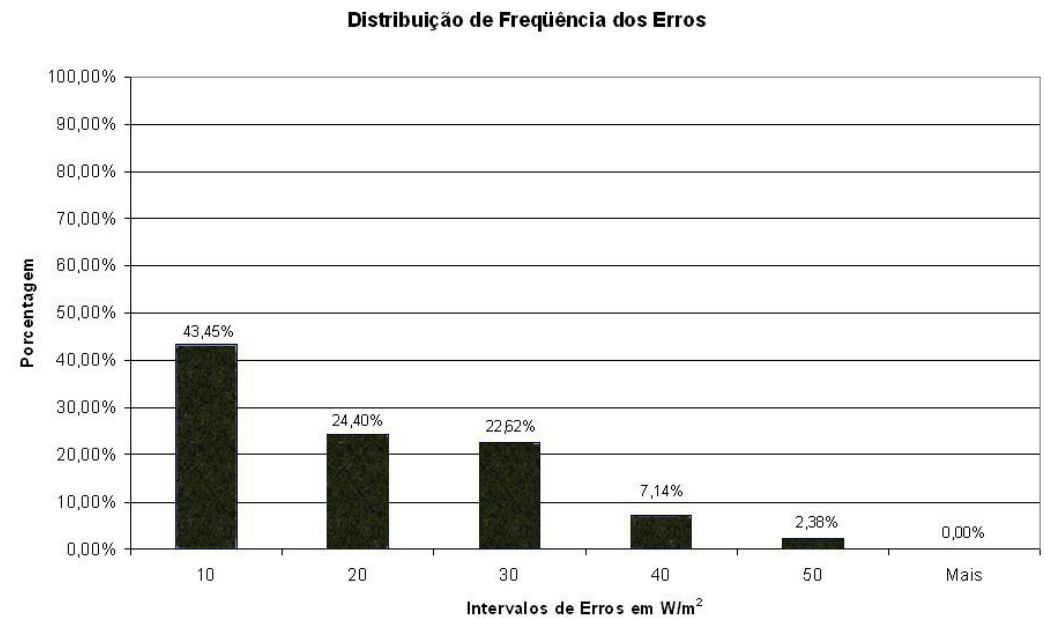

Figura 5 - Histograma de distribuição de freqüência dos erros absolutos entre as séries modeladas e observadas.

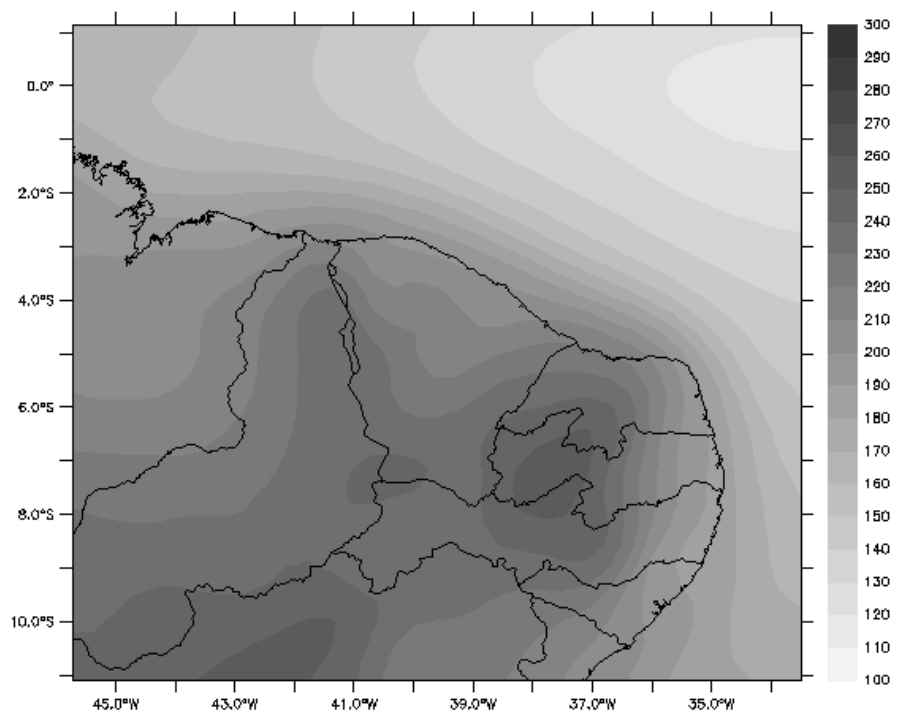

Figura 6 - Média anual corrigida através de regressão linear entre a fração de cobertura de nuvem e a razão entre a radiação observada sobre a radiação no topo da atmosfera. 
radiação solar global no estado do Ceará variam entre 200 e $250 \mathrm{~W} / \mathrm{m}^{2}$, com média de $215,3 \mathrm{~W} / \mathrm{m}^{2}$.

\section{SUMÁRIO E CONCLUSÕES}

O presente trabalho buscou apresentar uma metodologia para estimar a quantidade de radiação solar ao nível da superfície através do modelo atmosférico de mesoescala RAMS. Foram testadas, para o ano de 2005, as parametrizações de ChenCotton, 1983 e Harrington, 1997, usando, para validação dos resultados do modelo, os dados observados pelas Plataformas de Coleta de Dados (PCDs) da FUNCEME. Após validação, a parametrização de Chen-Cotton foi escolhida por seus resultados se aproximarem mais dos dados observados.

Embora o modelo não apresente destreza (skill) para estimar a radiação solar incidente diária, a média mensal da radiação solar incidente apresenta bons resultados. Porém, verificou-se que o modelo apresenta erros sistemáticos, superestimando a radiação solar, e por este motivo, alguns ajustes foram testados a fim de minimizar esses erros. Após estes ajustes, verificou-se uma boa correlação $(0,92)$ entre a fração de cobertura de nuvens e a razão entre a radiação observada na superfície e a radiação estimada no topo da atmosfera.

Para o estado do Ceará observou-se, nos resultados do modelo (após os ajustes), que a radiação solar incidente tem maiores médias durante o segundo semestre com um pico no mês de outubro $\left(260 \mathrm{~W} / \mathrm{m}^{2}\right)$, sendo o valor médio anual de 215 $\mathrm{W} / \mathrm{m} 2$. Estes valores obtidos são próximos aos apresentados no Atlas Brasileiro de Energia Solar (Martins et al., 2006).

Conclui-se, que a metodologia apresentada é bastante viável para estimar a quantidade de radiação solar ao nível da superfície em uma base mensal, podendo ser utilizada para diferentes resoluções espaciais, a critério do usuário e limitadas pelo custo computacional. A generalização dessa metodologia para uma série longa ( 30 anos) comporá a base de dados para a elaboração de um Atlas de Energia Solar para o Estado do Ceará (projeto em andamento).

\section{AGRADECIMENTOS}

Este trabalho recebeu suporte financeiro da Coordenação de Aperfeiçoamento de Pessoal de Nível Superior (CAPES) através de bolsa de mestrado para o primeiro autor e do Conselho Nacional de Desenvolvimento Científico e Tecnológico (CNPq), para infra-estrutura computacional da Fundação Cearense de Meteorologia e Recursos Hídricos - FUNCEME.

\section{REFERÊNCIAS BIBLIOGRÁFICAS}

CEBAllos, J.C., M.J. BotTino, J.M. de SOUZA. A simplified physical model for assessing solar radiation over Brazil using GOES 8 visible imagery. J. of Geophys. Research, v. 109, D02211, doi:10.1029/2003JD003531, 2004.

CHEN, Chaing; COTTON, William R. A one-dimensional simulation of the stratocumulus-capped mixed layer. Boundary-layer Meteorology, Boston, n. 25, p.289-321, 1983.

COTTON, W.R. et al. RAMS 2001: Current status and future directions. Meteor. Atmos Physics, n. 82, p.5-29, 2003.

GUARNIERI, Ricardo André et al. Previsões de Radiação Solar Utilizando Modelo de Mesoescala: Refinamento com Redes Neurais. In: CBENS - CONGRESSO BRASILEIRO DE ENERGIA SOLAR, 1., 2007, Fortaleza. Anais. Fortaleza: Abens - Associação Brasileira de Energia Solar, 2007. v. 1, CD-ROM.

HARRINGTON, J. The effects of radiative and microphysical processes on simulated warm and transition season arctic stratus. PhD. Colorado State University, 1997.

MARTINS, Fernando Ramos; ABREU, Samuel Luna de; RÜTHER, Ricardo. Atlas brasileiro de energia solar. São José Dos Campos: Inpe, 2006. 60 p.

PIELKE, R.A. et al.: A comprehensive meteorological modeling system - RAMS. Meteorol. Atmos. Phys., n. 49, p. 69-91, 1992

WALKO, R.L. et al. New RAMS cloud microphysics parameterization: Part I: the single-moment scheme. Atmospheric Research, n. 38, p.29-62, 1995. 